\title{
Glutathione status in critically-ill patients: possibility of modulation by antioxidants
}

\author{
Jan Wernerman*, Jia-Li Luo and Folke Hammarqvist \\ Department of Anaesthesia and Intensive Care and Unit of Anaesthesiologic Metabolism at KFC, Huddinge Hospital, \\ Department of Surgery, St Görans Hospital, Karolinska Institutet, S-141 86 Huddinge, Stockholm, Sweden
}

\begin{abstract}
Muscle tissue serves as a protein reservoir which is mobilized to meet the specific metabolic needs associated with various catabolic conditions in human subjects, such as trauma and critical illness. Glutathione is one of the most abundant short-chain peptides and a major source of non-protein thiol in the body, and tissue glutathione concentration is related to its oxidative capacity. Skeletal muscle is relatively unique with respect to a variety of metabolic properties, such as oxidative potential, patterns of amino acid utilization, and antioxidant enzyme activity. The glutathione concentration is not influenced by food intake, or by food deprivation. Moreover, there is no diurnal variation on muscle glutathione levels. Following elective surgery the muscle concentration of GSH (the reduced form) decreases by $40 \% 24 \mathrm{~h}$ post-operatively, while the concentration of GSSG (the oxidized form) remains unaltered. During critical illness a similar decrease in the GSH concentration is seen, but in addition a change in the redox status indicative of an elevated GSSG level occurs. Furthermore, correlations between the concentrations of glutamine as well as glutamate and GSH exist in these patients. From available evidence accumulated it is clear that glutathione plays a pivotal role in the maintenance of the intracellular redox status, the antioxidant vitamin levels, and the antioxidant enzyme functions under various metabolic conditions. The effectiveness of glutathione protection in the individual tissue depends on the tissue concentration of glutathione as well as the capacity of the tissue to import GSH and to export GSSG. The mechanisms by which catabolism regulates tissue glutathione levels and the enzyme activities associated with the $\gamma$-glutamyl cycle are not completely understood and further studies need to be conducted.
\end{abstract}

Glutathione: Critically ill: Glutamine: Skeletal muscle

The tripeptide glutathione (glycyl-glutamyl-cysteine) is in quantitative terms one of the most important antioxidants in human cells. Likewise, it has been suggested that glutathione plays an important role in the regulation of protein synthesis as well as protein degradation. Particularly in the liver, glutathione is also involved in detoxification and metabolism of a number of substances. In addition, glutathione is also involved in transmembrane transport of amino acids, in particular cysteine, which has traditionally been regarded as the limiting amino acid for glutathione metabolism as well as for protein synthesis (Meister \& Andersson, 1983; Deneke et al. 1989; Dolphin et al. 1989; Taniguchi et al. 1989).

Knowledge about the functions of glutathione has been closely linked to the accuracy of glutathione analyses. Being a thiol glutathione appears in an oxidized (GSSG) and a reduced (GSH) state. The state of oxidation is a matter of considerable controversy in the literature, as GSH and the total amount of glutathione are generally analysed but not GSSH. Thus, published results must be reviewed in the light of what analyses have been used in the particular study. There is a substantial literature concerning glutathione metabolism and tissue levels in mice and rats. In human subjects, on the other hand, the knowledge about glutathione is mostly confined to plasma and blood analyses. The importance of glutathione is mainly related to the clinical picture in individuals suffering from enzymic deficiencies in the biosynthesis of glutathione (Larsson, 1989).

The liver seems to be the central organ in glutathione metabolism, and plasma and erythrocyte concentrations are thought to reflect the synthetic capacity of the liver. In other organs the origin, biosynthesis and turnover of glutathione is 
more obscure (Adams et al. 1983; Meister \& Andersson, 1983; Lauterburg et al. 1984; DeLeve \& Kaplowitz, 1990).

\section{Biochemistry}

The first step in the synthesis of glutathione is the production of the dipeptide $\gamma$-glutamyl-cysteine from cysteine and glutamate. The reaction is catalysed by $\gamma$-glutamyl-cysteine synthase (EC 3.4.21.19). This step has traditionally been considered rate-limiting and the enzyme activity is regulated by feedback inhibition by glutathione. The second synthetic step is catalysed by glutathione synthase by adding glycine to the $\gamma$-glutamyl-cysteine dipeptide. In particular, the $\gamma$-glutamyl-cysteine synthase reaction is also involved in the transmembrane transport of the participating amino acids.

The degradation of glutathione is catalysed by $\gamma$-glutamyl transpeptidase (EC 2.3.2.2) and a number of dipeptidases bound to the external surface of the cell membrane. Normally GSH is not degraded intracellularly but is exported out of the cell. In contrast, rather small amounts of GSSG are transported out of the cell. However, in situations where there are increased intracellular levels of GSSG, the transmembrane export of GSSG is markedly increased (Srivastava \& Beutler, 1969; Akerboom \& Sies, 1994).

In addition to the synthesis and degradation of glutathione, the molecule undergoes changes between the reduced and oxidized state. The oxidation of GSH to GSSG is catalysed by glutathione peroxidase (EC 1.11.1.9), and while GSH is a monomer GSSG is a dimer. The GSH is predominant, normally constituting more than $90 \%$. Reduction of GSSG to GSH is catalysed by glutathione reductase (EC 1.6.4.2) using NADPH as the reducing agent. As a consequence of the increased extracellular leakage of GSSG in situations where GSSG is accumulated, GSSG formation leads to depletion of the intracellular pool of glutathione. Another pathway of glutathione depletion is via the reaction of GSSG with cellular proteins which are then degraded.

\section{Analytical techniques}

In biological samples specific enzymic reactions and chromatographic procedures are usually utilized for glutathione analyses. For determination of GSH, oxidation with 5'5'-dithiobis-2-nitrobenzoic acid produces GSSG with stoichiometric formation of 5-thiol-2-nitrobenzoic acid. The method depends on accurate standard curves and appropriate standards. A number of sensitive and specific chromatographic methods are also available, and recently, in particular with the application of HPLC, several rapid methods have come into use. We have adopted a technique based on the use of monobromobimane which has been adapted and developed to measure concentrations of glutathione, cysteine and their respective disulphides (Luo et al. 1995, 1998). Quantification of the bimane adducts of glutathione and cysteine is achieved by reversed-phase HPLC. Additionally, the method is coupled with the prederivatization procedures using dithiothreitol and N-ethylmaleimide for the measurement of the thiol disulfides. Full validation results for quantitative measurements have been conducted using acid extracts of muscle tissue, with high analytical recoveries of $97 \%$ for GSH and $>92 \%$ for GSSG, and also within-run variations of $<3 \%$ and between-run variations of $<10 \%$. This method based on monobromobimane labelling offers the broadest range of application for thiol determination; however, this method cannot be used to measure glutathione derivatives in which the thiol group is blocked.

\section{Glutathione and glutamine}

In muscle, free glutamine plays a central role in amino acid metabolism. It is produced at a high rate and exported from muscle mainly to organs or cells in the splanchnic area. Free glutamine is maintained at a very high concentration in skeletal muscle and a significant correlation $(r 0.87$; $P<0.001)$ between glutamine concentration and muscle protein synthesis has been reported in malnourished and endotoxaemic rats (Jepson et al. 1988). In human subjects, a significant correlation $(P<0 \cdot 01)$ between the change in glutamine and the change in protein synthesis was found following surgical trauma (Wernerman et al. 1990). Furthermore, a correlation between glutamine and protein degradation has also been reported in an experimental rat muscle system (MacLennan et al. 1988). As free glutamine and free glutamate concentrations in muscle are closely linked, we became interested in the possible relationship between glutamine and glutathione. In the liver, glutamine supplementation has been shown to benefit glutathionedepleted animals (Hong et al. 1992; Robinson et al. 1992). Although a significant relationship between protein metabolism and glutamine concentration in muscle has not been established, at least in some situations the interaction between glutamine and protein metabolism can be regulated possibly via glutathione. As animal experiments have shown that glutathione concentration is at the mmol level in several tissues, a study was initiated to investigate glutathione in human skeletal muscle.

\section{Glutathione in human muscle}

In the post-absorptive state GSH concentration in human muscle is 1.40 (SD 0.20) $\mathrm{mmol} / \mathrm{kg}$ wet weight. The muscle GSH concentration in young healthy male volunteers is similar to that in non-depleted patients up to the age of 80 years undergoing elective surgery (Luo et al. 1995, 1999). The muscle GSH concentration does not undergo diurnal variations or respond to feeding, but stays constant in normal human subjects in the fed state (Luo et al. 1995). Furthermore, short-term starvation for $72 \mathrm{~h}$, which causes a dramatic reduction in muscle free glutamine, is not associated with any change in muscle GSH (Andersson et al. 1994).

Elective abdominal surgery, which causes a 30-40\% reduction in muscle free glutamine, is accompanied by a $40 \%$ reduction in muscle glutathione (Luo et al. 1996, 1998). The reduction is predominantly in GSH, while GSSG is proportionally the same. The maximal decrease is seen at $24 \mathrm{~h}$ after surgery, and at $72 \mathrm{~h}$ an increase towards restitution is observed, although at that time the concentration is not completely normalized. When studying enzymic activities, the activity of glutathione synthase appears to be reduced in 
this situation. This observation suggests a different regulation of glutathione homeostasis in human muscle compared with rat liver. The time course of the decrease in GSH coincides with the decrease in glutamate, but comes a little earlier than the decrease in glutamine. Following major vascular surgery, including up to $90 \mathrm{~min}$ of ischaemia in the legs, there is a pattern similar to that seen after abdominal surgery (Westman et al. 1998). Also, after maximal ischaemia, and immediately after reperfusion, glutathione levels are only marginally influenced. Later a decrease is observed, but the contribution of surgical trauma itself and that of ischaemia in this particular patient model cannot be separated. This finding fits well with the clinical practise of restricting ischaemia to less than 90 min during vascular surgery.

In intensive-care (ICU) patients with septic complications following surgery, muscle free glutamine is depleted to less than $25 \%$ of the normal concentration (Hammarqvist et al. 1997). In parallel, during the first week in ICU muscle GSH concentration is reduced to the same level or greater compared with patients undergoing elective surgery. Among ICU patients the scatter in muscle GSH concentration is more pronounced as compared with post-operative patients. Another difference is that GSSG maintains a high concentration both in absolute and relative terms. Furthermore, in this situation there is a significant correlation between muscle free glutamine and muscle total glutathione concentration as well as GSSG : total glutathione $\left(r^{2} 0.46\right.$ and 0.42 ; $P<0.001$ respectively). However, when consecutive biopsies were studied in a small group of ICU patients a tendency towards normalization of the total glutathione level was observed. Needless to say, this observation needs to be confirmed in a larger sample of patients (Fläring et al. 1999).

\section{Other human tissues}

As the information on glutathione concentrations in human tissues is not extensive, we have also studied other tissues in the post-absorptive state. Liver and duodenal mucosa maintain a concentration two to three times that of skeletal muscle (3.69 (SD 0.78) and 4.47 (SD 0.41) $\mathrm{mmol} / \mathrm{kg}$ wet weight respectively). Also, the gastric mucosa has a higher concentration than that in muscle $(2.49$ (SD 0.33) $\mathrm{mmol} / \mathrm{kg}$ wet weight). All these tissues have a small proportion of GSSG which is of similar magnitude to that in muscle. Whole blood has a glutathione concentration of 0.57 (SD $0.06) \mathrm{mmol} / \mathrm{l}$ and the glutathione is almost totally confined to the blood cells, as the plasma concentration is only 4.9 (SD 1.8) $\mu \mathrm{mol} / \mathrm{l}$.

\section{Concluding remarks}

The possible relationship between glutamine and glutathione has been shown as a correlation between the respective depletions observed in ICU patients. Recent studies have also shown that glutamine supplementation is protective after severe injury through the preservation of tissue glutathione stores (Hong et al. 1992). In an animal model of induced hepatic necrosis, the availability of glutamine appears to be critical for the generation of glutathione stores by providing the glutamate portion of glutathione. Since glutamine is efficiently transported across cell membranes, it may be a readily-available precursor for glutathione synthesis. Evidence supporting this hypothesis includes: (1) radiolabelling studies in erythrocytes and kidney have shown that the glutamate portion of glutathione is derived preferentially from glutamine (Miller \& Horiuchi, 1963; Cook \& Peters, 1986); (2) hepatocytes cultured in a glutamine-free medium were rapidly depleted of glutamate, resulting in decreased intracellular glutathione levels (Taniguchi et al. 1989); (3) inhibition of glutamine transport into cultured lymphocytes resulted in a decrease in intracellular glutathione in a dose-dependent manner (Novogrodsky et al. 1979); (4) in isolated perfused kidney under specific conditions, glutamine was found to be ratelimiting for glutathione synthesis (Welbourne, 1979). Glutamine supplementation enhanced plasma GSH concentration, and preserved GSH levels during intestinal ischaemia and reperfusion (Harward et al. 1994; Denno et al. 1996). Thus, it is possible that intravenous glutamine supplementation is a simple, safe and relatively inexpensive method of enhancing antioxidant protection.

In summary, although the role of glutathione in cellular antioxidant defence has been well established, its significance in protecting skeletal muscle tissue against free radical-induced oxidative stress and tissue damage following surgical trauma and critical illness has not been investigated until recently. From available evidence, it has become increasingly clear that glutathione plays a pivotal role in the maintenance of intracellular redox status, antioxidant vitamin levels, and antioxidant enzyme functions under various metabolic conditions. The effectiveness of glutathione protection in the various tissues depends on the concentration of tissue glutathione as well as the capacity of the tissue to import GSH and to export GSSG. The possible mechanisms by which catabolism regulates tissue glutathione levels and the enzyme activities associated with the $\gamma$-glutamyl cycle are not completely understood and further studies need to be conducted.

\section{References}

Adams JD, Lauterburg BH \& Mitchell JR (1983) Plasma glutathione and glutathione disulfide in the rat: regulation and response to oxidative stress. Journal of Pharmacology and Experimental Therapeutics 227, 749-754.

Akerboom TP \& Sies H (1994) Transport of glutathione disulfide and glutathione mixed disulfides in biological samples. Methods in Enzymology 77, 373-382.

Andersson K, Luo J-L, Hammarqvist F \& Wernerman J (1994) The effect of fasting on muscle glutathione and amino acid levels. Clinical Nutrition 13, Suppl., 4-5.

Cook ND \& Peters TJ (1986) The simultaneous hydrolysis of glutathione and glutamine by rat kidney gamma-glutamyl transferase. Biochimica et Biophysica Acta 884, 207-210.

DeLeve LD \& Kaplowitz N (1990) Importance and regulation of hepatic glutathione. Seminars in Liver Disease 10, 251-266.

Deneke SM, Baxter DF, Phelps DT \& Fanburg BL (1989) Increase in endothelial cell glutathione and precursor amino acid uptake by ethyl maleate and hypoxia. American Journal of Physiology 275, L265-L271.

Denno R, Rounds JD, Faris R, Holejko LB \& Wilmore DW (1996) Glutamine-enriched total parenteral nutrition enhances plasma 
glutathione in resting state. Journal of Surgical Research 61, 35-38.

Dolphin D, Poulson R \& Avramovic O (editors) (1989) Glutathione: Chemical, Biochemical and Medical Aspects. New York: Wiley.

Fläring U, Wernerman J \& Hammarqvist F (1999) Muscle glutathione concentrations in critically ill patients. Clinical Nutrition (In the Press).

Hammarqvist F, Luo J-L, Andersson K, Cotgreave IA \& Wernerman J (1997) Skeletal muscle glutathione is depleted in critically ill patients. Critical Care Medicine 25, 78-84.

Harward TRS, Coe D, Souba WW, Klingman N \& Seeger JM (1994) Glutamine preserves gut glutathione levels during intestinal ischemia/reperfusion. Journal of Surgical Research 56, 351-355.

Hong RW, Rounds JD, Helton WS, Robinson MK \& Wilmore DW (1992) Glutamine preserves liver glutathione and improves survival after lethal hepatic injury. Annals of Surgery 215, 114-119.

Jepson MM, Bates PC, Broadbent P, Pell JM \& Millward DJ (1988) Relationship between glutamine concentration and protein synthesis in rat skeletal muscle. American Journal of Physiology 255, E166-E172.

Larsson A (1989) Hereditary disorders related to glutathione deficiency. In Glutathione: Chemical, Biochemical, and Medical Aspects, pp. 197-234 [D Dolphin, O Avramovic and R Poulson, editors]. New York: Wiley.

Lauterburg BH, Adams JD \& Mitchell JR (1984) Hepatic glutathione homeostasis in the rat: efflux accounts for glutathione turnover. Hepatology 4, 586-590.

Luo J-L, Hammarqvist F, Andersson K \& Wernerman J (1996) Skeletal muscle glutathione following surgical trauma. Annals of Surgery 223, 420-427.

Luo J-L, Hammarqvist F, Andersson K \& Wernerman J (1998) Surgical trauma decreases the synthesis of glutathione in skeletal muscle. American Journal of Physiology 275, E359-E365.

Luo J-L, Hammarqvist F, Cotgreave IA, Lind K, Andersson K \& Wernerman J (1995) Determination of intracellular glutathione in human skeletal muscle by reversed-phase high-performance liquid chromatography. Journal of Chromatography 670B, 29-36.

Luo J-L, Hammarqvist F, Lindholmer C, Andersson K \& Wernerman J (1999) Determination of the redox status of glutathione and cysteine in small biopsy specimens of human tissue. Clinical Chemistry (In the Press).

MacLennan PA, Smith K, Weryk B, Watt PW \& Rennie MJ (1988) Inhibition of protein breakdown by glutamine in perfused rat skeletal muscle. FEBS Letters 237, 133-136.

Meister A \& Andersson ME (1983) Glutathione. Annual Review of Biochemistry 52, 711-760.

Miller A \& Horiuchi M (1963) Erythrocyte GSH. In vitro incorporation of radioactive amino acid precursors into the GSH of human erythrocytes. Journal of Laboratory and Clinical Medicine 60, 764-765.

Novogrodsky A, Nehring RE \& Meister A (1979) Inhibition of amino acid transport into lymphoid cells by the glutamine analog L-2-amino-4oxo-5-chloropentanoate. Proceedings of the National Academy of Sciences, USA 76, 4932-4935.

Robinson MK, Rounds JD, Hong RW, Jacobs DO \& Wilmore DW (1992) Glutathione deficiency increases organ dysfunction after hemorrhagic shock. Surgery 112, 140-149.

Srivastava SK \& Beutler E (1969) The transport of oxidised glutathione from human erythrocytes. Journal of Biological Chemistry 244, 9-16.

Taniguchi N, Higashi T, Sakamoto Y \& Meister A (1989) Glutathione Centennial: Molecular Properties and Clinical Implications. New York: Academic Press.

Welbourne TC (1979) Ammonia production and glutamine incorporation into glutathione in the functioning rat kidney. Canadian Journal of Biochemistry 57, 233-237.

Wernerman J, Hammarqvist F \& Vinnars E (1990) Alphaketoglutarate and postoperative muscle catabolism. Lancet 335, 701-703.

Westman B, Hammarqvist F, Johansson G, Luo J-L, Söderlund K \& Wernerman J (1998) Effects of ischemia and reperfusion on glutathione, energy and amino acid content in muscle following elective surgery of abdominal aneurysm. Hygea 197, 220 Abstr. 